\title{
Model for the Infrastructure of Engineering Education
}

\author{
James G. Harris
}

Win Aung
Karen Frair

Susan T. Hill
Introduction

There are many ways to describe and model physical systems. The infrastructure of engineering education in the United States is an example of such a system, and it is the intent of this report to describe and model this infrastructure. The means for doing this starts with the graduation statistics for the year 1989 given by the Computer Aided Science Policy Analysis \& Research Database System (CASPAR) developed for the National Science Foundation by Quantum Research Corporation [1], and uses a simple categorization for the institutions that contribute to those statistics. This categorization sorts the post-secondary institutions in the United States which enroll engineering students into three types: community colleges (or two year institutions), non-PhD granting institutions (Bachelors and/or Masters engineering degree granting), and $\mathrm{PhD}$ engineering degree granting institutions. The intent is to ascertain the contribution of each type of institution to the infrastructure of engineering education.

The National Model

Figure 1 presents the distribution of engineering degrees in the United States based upon the data given in Table I for the engineering PhDs and Bachelors degrees conferred in 1989 [1]. This data lists by academic institution the number of $\mathrm{PhD}$ and Bachelor degrees granted, with the institutions sorted first by the number of PhD degrees granted, and second by the number of Bachelor degrees for the non-PhD institutions. Columns in Table I also present the cumulative number of degrees and their corresponding cumulative percent of the total number of degrees. For example, MIT is indexed " 1 " for the PhD granting institutions with $217 \mathrm{PhD}$ degrees, and it granted 633 Bachelor degrees; and the University of Arizona is indexed "33" with 41 engineering $\mathrm{PhD}$ degrees and 562 engineering Bachelor degrees. For the non-PhD institutions, California Poly St U, San Luis Obispo is indexed first in the second sort criteria, or "163" in the total list, with 562 engineering Bachelor degrees; and, Bucknell University is indexed "209" overall with 116 engineering Bachelor degrees.

The curves in Figure 1 plot the cumulative percent of the total degrees for both PhDs and Bachelors presented in the Table as a function of the index of the academic institution. For example, at index " 40 ", University of California-Davis, $68 \%$ of the PhD degrees have been conferred, and $35 \%$ of the Bachelor degrees have been conferred; or put another way, the top $40 \mathrm{PhD}$ granting institutions graduate $68 \%$ of the PhD students, but only $35 \%$ of the Bachelor degree students. Note that for the $162 \mathrm{PhD}$ granting institutions, approximately two-thirds of the degrees are obtained at about one-quarter of the institutions. Nationally, $76 \%$ of all engineering Bachelor degrees are granted by the $\mathrm{PhD}$ granting institutions.
National data must be interpreted properly for the various states to prevent drawing erroneous conclusions. In Figure 2 the data for the state of California is plotted using the data in Table II. The data in the Table is presented using the same process as the national data, i.e., institutions are indexed first by the number $\mathrm{PhD}$ degrees conferred and then by the number of Bachelor degrees awarded by the non-PhD granting institutions. Table II is just a subset from the data in Table $I$, and, in principle, the data for each state can be plotted in an analogous fashion. In California, the $\mathrm{PhD}$ granting institutions only award $42 \%$ of the Bachelor degrees with the non-PhD granting institutions graduating $58 \%$ of the Bachelor degrees. The reason for this difference with the national curve for the Bachelor degrees can be attributed to the policy that the state initiated in the early 60 's, called the "Master Plan", which created a three-tired system of public higher education based upon the community colleges, the California State University, and the University of California. Following the same process, figures and tables are presented for Florida, Illinois, Massachusetts, Montana, New York, and Texas. Clearly, each state has its own characteristic.

\section{Community College Contributions}

Information on the contribution of community colleges to the graduates in engineering is difficult to acquire at this time. Nationally, it is estimated that in $1987,25 \%$ of the engineering Bachelor degree students transferred one or more courses to their graduating institution from a community college [2]. Therefore, to obtain precise information, a simple model is proposed. Logically, there are three possibilities for a Bachelor degree graduate to enter the institution from which they received their degree: 1) as a Freshman, 2) as a transfer student from another four year institution, and 3) as a transfer student from a community college. Using this model, information was requested from at least a dozen states to determine the percentage of Bachelor degrees students in engineering who entered their graduating institution by transferring from a community college. While all the states contacted were enthusiastic about obtaining the data, only a few states could provide preliminary data addressing the request.

Florida estimated that in $1989-90,14.6 \%$ of the graduates in engineering from the nine public-supported universities transferred from community colleges [3]. Califomia estimates that $24 \%$ of the University of California and $47 \%$ of the Califomia State University Bachclor degrees in engineering in 1985-86 resulted from transfer students from community colleges [4]. Washington estimates that about $30 \%$ of the engineering graduates from the University of Washington in 1990-91 
transferred from community colleges [5]. Lastly, Colorado estimates that at least $3.8 \%$ of the engineering graduates from the six public-supported engineering institutions transferred from. community colleges

Conclusion

In summary, it is hoped that this presentation will give some insight into the nature of the infrastructure of engineering education, nationally as well as for each state. The data has many interpretations, and this report should initiate much discussion regarding the mission of each institution and its contribution to the infrastructure of engineering education in the United States.
Bibliography

1. CASPAR data: Data collected by the National Center for Education Statistics, U.S. Department of Education, Completions Survey, 1989

2. Preliminary data provided by Elizabeth Teles, AACJC Fellow at the National Science Foundation, 6 July 1992

3. Telephone conversation with Joan Roddenberry, Division of Community Colleges of Florida, 12 July 1991

4. 1988 California Post-Secondary Education Commission report entitled "Update of California Transfer Students Fall 1987" (thanks to Jim Palmer, Center for Community College Education, George Mason University, in telephone conversation on 6 June 1991)

5. Data provided by Geogory P. Smith, Coordinator of Institutional Research and Education, Colorado Community College \& Occupational Education System on 21 April 1992 (thanks to Lourdes Tinajero, AACJC Fellow at the National Science Foundation)

Table I

Englneering Ph.D.s and Bachelor's Degrees Conferred in the United States, by Institution: 1989

\begin{tabular}{|c|c|c|c|c|c|c|c|}
\hline Academic Institution & PhDs & Bach & Cum & Cum & Rank & Cum PhD & Cum Bach \\
\hline & & & PhDS & Bach & & 0 & 0 \\
\hline Massachusetts Institute of Technology & 217 & 633 & 217 & 633 & 1 & 5 & 1 \\
\hline University of Callfornia-Berkeley & 201 & 517 & 418 & 1,150 & 2 & 9 & 2 \\
\hline Stanford University & 173 & 273 & 591 & 1,423 & 3 & 13 & 2 \\
\hline Unlversity of Illinois-Urbana & 136 & 1,259 & 727 & 2,682 & 4 & 16 & 4 \\
\hline Purdue University-AIl Campuses & 132 & 1,221 & 859 & 3,903 & 5 & 19 & 6 \\
\hline University of Michigan & 122 & 1,006 & 981 & 4,909 & 6 & 22 & 7 \\
\hline University of Texas-Austin & 107 & 647 & 1,088 & 5,556 & 7 & 24 & 8 \\
\hline Texas $A \& M$ University-AII Campuses & 98 & 1,013 & 2,186 & 6,569 & 8 & 26 & 10 \\
\hline University of Wisconsin-Madison & 98 & 836 & 1,284 & 7,405 & 9 & 28 & 11 \\
\hline Ohio State University-All Campuses & 97 & 702 & 1,381 & 8,107 & 10 & 30 & 12 \\
\hline Virginda Polytechnic Inst \& State Univ & 97 & 862 & 1,478 & 8,969 & 11 & 33 & 13 \\
\hline Pennsylvania State University-All Cam & 95 & 1,101 & 1,573 & 10,070 & 12 & 35 & 15 \\
\hline Cornell University & 88 & 568 & 1,661 & 10,638 & 13 & 37 & 16 \\
\hline University of Southern Californta & 80 & 319 & 1,741 & 10,957 & 14 & 38 & 16 \\
\hline Georgla Inst1tute of Technology-All Cam & 78 & 1,015 & 1,819 & 11,972 & 15 & 40 & 18 \\
\hline Carnegie-Mellon University & 76 & 277 & 1,895 & 12,249 & 16 & 42 & 18 \\
\hline North Carolina State University-Ralelgh & 76 & 924 & 1,971 & 13,173 & 17 & 43 & 20 \\
\hline University of Minnesota & 75 & 724 & 2,046 & 13,897 & 18 & 45 & 21 \\
\hline Case western Reserve University & 74 & 256 & 2,120 & 14,153 & 19 & 47 & 21 \\
\hline Northwestern University & 74 & 250 & 2,194 & 14,403 & 20 & 48 & 21 \\
\hline Rensselaer Polytechnic Institute & 73 & 618 & 2,267 & 15,021 & 21 & 50 & 22 \\
\hline University of Florida & 60 & 583 & 2,327 & 15,604 & 22 & 51 & 23 \\
\hline University of Californda-Los Angeles & 59 & 327 & 2,386 & 15,931 & 23 & 53 & 24 \\
\hline University of Maryland-College Park & 57 & 660 & 2,443 & 16,591 & 24 & 54 & 25 \\
\hline Iowa State University of Sclence $\&$ Tech & 56 & 791 & 2,499 & 17,382 & 25 & 55 & 26 \\
\hline University of Colorado & 47 & 631 & 2,546 & 18,013 & 26 & 56 & 27 \\
\hline University of Washington & 45 & 616 & 2,591 & 18,629 & 27 & 57 & 28 \\
\hline University of Massachusetts system & 43 & 418 & 2,634 & 19,047 & 28 & 58 & 28 \\
\hline Columbla Unlversity & 42 & 238 & 2,676 & 19,285 & 29 & 59 & 29 \\
\hline University of Pennsylvania & 42 & 237 & 2,718 & 19,522 & 30 & 60 & 29 \\
\hline Princeton University & 41 & 165 & 2,759 & 19,687 & 31 & 61 & 29 \\
\hline SUNY-Buffalo & 41 & 525 & 2,800 & 20,212 & 32 & 62 & 30 \\
\hline University of Arizona & 41 & 562 & 2,841 & 20,774 & 33 & 63 & 31 \\
\hline California Institute of Technology & 39 & 147 & 2,880 & 20,921 & 34 & 63 & 31 \\
\hline Lehigh University & 38 & 388 & 2,918 & 21,309 & 35 & 64 & 32 \\
\hline Michigan State University & 38 & 529 & 2,956 & 21,838 & 36 & 65 & 32 \\
\hline University of Utah & 37 & 290 & 2,993 & 22,128 & 37 & 66 & 33 \\
\hline Arizona State University & 35 & 453 & 3,028 & 22,581 & 38 & 67 & 34 \\
\hline
\end{tabular}




\begin{tabular}{|c|c|c|c|c|c|c|c|}
\hline Colorado State Universicy & 35 & 248 & 3,063 & 22,829 & 39 & 68 & 34 \\
\hline University of Callfornla-Davig & 35 & 372 & 3,098 & 23,201 & 40 & 68 & 35 \\
\hline University of virginia & 33 & 245 & 3,131 & 23,446 & 41 & 69 & 35 \\
\hline University of Houston & 32 & 202 & 3,163 & 23,648 & 42 & 70 & 35 \\
\hline Rutgers State Unfversity of New Jersey & 31 & 452 & 3,194 & 24,100 & 43 & 70 & 36 \\
\hline University of Pittsburgh & 31 & 387 & 3,225 & 24,487 & 44 & 71 & 36 \\
\hline University of Missouri-Columbia & 28 & 433 & 3,253 & 24,920 & 45 & 72 & 37 \\
\hline George Washington University & 27 & 171 & 3,280 & 25,091 & 46 & 72 & 37 \\
\hline Johns Hopkins University & 27 & 181 & 3,307 & 25,272 & 47 & 73 & 38 \\
\hline Rice University & 27 & 145 & 3,334 & 25,417 & 48 & 74 & 38 \\
\hline University of California-San Diego & 27 & 284 & 3,361 & 25,701 & 49 & 74 & 38 \\
\hline Unlversity of Cincinnati-All Campuses & 26 & 404 & 3,387 & 26,105 & 50 & 75 & 39 \\
\hline University of Delaware & 26 & 203 & 3,413 & 26,308 & 51 & 75 & 39 \\
\hline University of IIIinols-chlcago & 26 & 411 & 3,439 & 26,719 & 52 & 76 & 40 \\
\hline Clarkson University. & 25 & 659 & 3,464 & 27,378 & 53 & 76 & 41 \\
\hline Clemson University & 25 & 505 & 3,489 & 27,883 & 54 & 77 & 41 \\
\hline Oxlahoma State University & 25 & 314 & 3,514 & 28,197 & 55 & 77 & 42 \\
\hline University of Missouri-Rolla & 25 & 737 & 3,539 & 28,934 & 56 & 78 & 43 \\
\hline Drexel University & 24 & 544 & 3,563 & 29,478 & 57 & 79 & 44 \\
\hline Syracuse University-All Campuses & 24 & 239 & 3,587 & 29,717 & 58 & 79 & 44 \\
\hline Duke University & 23 & 171 & 3,610 & 29,888 & 59 & 80 & 44 \\
\hline University of Callfornia-Santa Barbara & 23 & 194 & 3,633 & 30,082 & 60 & 80 & 45 \\
\hline University of Rhode Island & 23 & 184 & 3,656 & 30,266 & 61 & 81 & 45 \\
\hline Auburn University-All Campuses & 22 & 820 & 3,678 & 31,086 & 62 & 81 & 46 \\
\hline University of Notre Dame & 22 & 279 & 3,700 & 31,365 & 63 & 82 & 47 \\
\hline Oregon State University & 21 & 314 & 3,721 & 31,679 & 64 & 82 & 47 \\
\hline Southern Methodist University & 21 & 91 & 3,742 & 31,770 & 65 & 82 & 47 \\
\hline Univergity of California-Irvine & 21 & 181 & 3,763 & 31,951 & 66 & 83 & 48 \\
\hline Untverstty of Iowa & 21 & 221 & 3,784 & 32,172 & 67 & 83 & 48 \\
\hline University of Oxlahoma & 21 & 247 & 3,805 & 32,419 & 68 & 84 & 48 \\
\hline University of Tennessee-Knoxvilie & 21 & 447 & 3,826 & 32,866 & 69 & 84 & 49 \\
\hline Brigham Young University-AII Campuses & 20 & 287 & 3,846 & 33,153 & 70 & 85 & 49 \\
\hline University of New Mexico & 20 & 231 & 3,866 & 33,384 & 71 & 85 & 50 \\
\hline Polytechnic University & 19 & 413 & 3,885 & 33,797 & 72 & 86 & 50 \\
\hline University of Connecticut & 19 & 273 & 3,904 & 34,070 & 73 & 86 & 51 \\
\hline Illinois Instituta of Technology & 18 & 314 & 3,922 & 34,384 & 74 & 86 & 51 \\
\hline University of Texis-Arlington & 18 & 340 & 3,940 & 34,724 & 75 & 87 & 52 \\
\hline Yale University & 18 & 27 & 3,958 & 34,751 & 76 & 87 & 52 \\
\hline University of Akron-All Campuses & 17 & 259 & 3,975 & 35,010 & 77 & 88 & 52 \\
\hline Undversity of Kansas & 17 & 283 & 3,992 & 35,293 & 78 & 88 & 53 \\
\hline West Virginia University & 17 & 225 & 4,009 & 35,518 & 79 & 88 & 53 \\
\hline Texas Tech Univer:ity & 16 & 243 & 4,025 & 35,761 & 80 & 89 & 53 \\
\hline Vanderbilt University & 16 & 205 & 4,041 & 35,966 & 81 & 89 & 54 \\
\hline Washington University & 16 & 183 & 4,057 & 36,249 & 82 & 89 & 54 \\
\hline Stevens Institute of Technologr & 15 & 233 & 4,072 & 36,382 & 83 & 90 & 54 \\
\hline SUNY-stony Brook & 15 & 187 & 4,087 & 36,569 & 84 & 90 & 54 \\
\hline University of Idaho & 15 & 166 & 4,102 & 36,735 & 85 & 90 & 55 \\
\hline University of Rochester & 15 & 179 & 4,117 & 36,914 & 86 & 91 & 55 \\
\hline Loulsiana State University-AlI Campuses & 14 & 285 & 4,131 & 37,199 & 87 & 91 & 55 \\
\hline New Jersey Inst1tute of Technolagy & 14 & 417 & 4,145 & 37,616 & 88 & 91 & 56 \\
\hline Untversity of Kentucky & 14 & 220 & 4,159 & 37,836 & 89 & 92 & 56 \\
\hline Colorado School of Mines & 13 & 274 & 4,172 & 38,110 & 90 & 92 & 57 \\
\hline Utah State University & 13 & 121 & 4,185 & 38,231 & 91 & 92 & 57 \\
\hline Wichita State University & 13 & 162 & 4,198 & 38,393 & 92 & 93 & 57 \\
\hline University of Alabama & 12 & 245 & 4,210 & 38,638 & 93 & 93 & 57 \\
\hline Wayne State University & 12 & 179 & 4,222 & 38,817 & 94 & 93 & 58 \\
\hline old Dominion University & 11 & 205 & 4,233 & 39,022 & 95 & 83 & 58 \\
\hline University of Toledo & 11 & 288 & 4,244 & 39,310 & 96 & 94 & 58 \\
\hline Erown University & 10 & 87 & 4,254 & 39,397 & 97 & 94 & 59 \\
\hline Univezsity of Nebraska-Itrooln & 10 & 241 & 4,264 & 39,638 & 98 & 34 & 59 \\
\hline Washington State Oriversity & 10 & 363 & 4,274 & 40,001 & 99 & 94 & 60 \\
\hline worcester Poiytechnic Institute & 10 & 408 & 4,284 & 40,409 & 100 & 94 & 50 \\
\hline Joulstana pech ontrersity & 9 & 192 & 4,293 & 40,602 & 101 & 95 & 60 \\
\hline Mississipei state batrexsity & 9 & 328 & 4,302 & 40,929 & 102 & 95 & E: \\
\hline Northeastern Untvers? & 9 & 583 & 4,311 & 43,522 & 103 & 95 & 62 \\
\hline Univezsity of Alabama-Hintsvilae & 9 & 199 & 4,320 & 41,711 & 104 & 95 & 62 \\
\hline University of Nians (FI) & 9 & 266 & 4,329 & 41,977 & 105 & 95 & 62 \\
\hline Univereity of south Canofine-hil Campuse & 3 & 202 & 4,339 & 42,279 & 206 & 96 & 63 \\
\hline CUNI-Graduste sonool and Undversity cits & 8 & 0 & 4,346 & 42,279 & 207 & 96 & $\epsilon 3$ \\
\hline Konsas state Unirezsity of ha a hppl Sci & 8 & 390 & 4,354 & 42,569 & 108 & 96 & 63 \\
\hline Her Mexico state university-hil Campuses & 8 & 242 & 4,352 & 42,811 & 109 & 96 & 64 \\
\hline
\end{tabular}




\begin{tabular}{|c|c|c|c|c|c|c|c|}
\hline University of Dayton & 8 & 160 & 4,370 & 42,971 & 110 & 96 & 64 \\
\hline Catholic University of America & 7 & 91 & 4,377 & 43,062 & 111 & 96 & 64 \\
\hline Michigan Technological University & 7 & 738 & 4,384 & 43,800 & 112 & 97 & 65 \\
\hline University of Mississippi & 7 & 63 & 4,391 & 43,863 & 113 & 97 & 65 \\
\hline University of Wyoming & 7 & 183 & 4,398 & 44,046 & 114 & 97 & 66 \\
\hline Tennessee Technological University & 6 & 301 & 4,404 & 44,347 & 115 & 97 & 66 \\
\hline Undversity of Arkansas-Fayetteville & 6 & 287 & 4,410 & 44,634 & 116 & 97 & 66 \\
\hline Universitz of South Florida & 6 & 380 & 4,416 & 45,014 & 117 & 97 & 67 \\
\hline Alfred Univers1t Y Main Campus & 5 & 89 & 4,421 & 45,103 & 118 & 97 & 67 \\
\hline Cleveland State University & 5 & 188 & 4,426 & 45,291 & 119 & 98 & 67 \\
\hline Florida Atlantic University & 5 & 143 & 4,431 & 45,434 & 120 & 98 & 68 \\
\hline Instltute of Paper Chemistry & 5 & 0 & 4,436 & 45,434 & 121 & 98 & 68 \\
\hline Ohio University-All Campuses & 5 & 206 & 4,441 & 45,640 & 122 & 98 & 68 \\
\hline Tulane University of Loulsiana & 5 & 151 & 4,446 & 45,791 & 123 & 98 & 68 \\
\hline University of Maine-orono & 5 & 201 & 4,451 & 45,992 & 124 & 98 & 68 \\
\hline University of North Carolina-Chapel Hill & 5 & 0 & 4,456 & 45,992 & 125 & 98 & 68 \\
\hline Harvard University & 4 & 27 & 4,460 & 46,019 & 126 & 98 & 68 \\
\hline Oregon Graduate Center & 4 & 0 & 4,464 & 46,019 & 127 & 98 & 68 \\
\hline Tufts University & 4 & 158 & 4,468 & 46,177 & 128 & 99 & 69 \\
\hline University of Loulsuille & 4 & 203 & 4,472 & 46,380 & 129 & 99 & 69 \\
\hline Air Force Institute of Technology & 3 & 0 & 4,475 & 46,380 & 130 & 99 & 69 \\
\hline Boston Unfversity & 3 & 385 & 4,478 & 46,765 & 131 & 99 & 70 \\
\hline Florida Insitute of Technology & 3 & 237 & 4,481 & 47,002 & 132 & 99 & 70 \\
\hline New Mexico Institute of Mining \& Technol & 3 & 48 & 4,484 & 47,050 & 133 & 99 & 70 \\
\hline North Dakota State University-AIl Cam & 3 & 346 & 4,487 & 47,396 & 134 & 99 & 71 \\
\hline Oakland University & 3 & 124 & 4,490 & 47,520 & 135 & 99 & 71 \\
\hline Portland State UnIversity & 3 & 132 & 4,493 & 47,652 & 136 & 99 & 71 \\
\hline University of Detroit & 3 & 108 & 4,496 & 47,760 & 137 & 99 & 71 \\
\hline University of Tulsa & 3 & 137 & 4,499 & 47,897 & 138 & 99 & 71 \\
\hline University of vermont & 3 & 127 & 4,502 & 48,024 & 139 & 99 & 71 \\
\hline Albany Law School Union Univergity & 2 & 0 & 4,504 & 48,024 & 140 & 99 & 71 \\
\hline Howard University & 2 & 108 & 4,506 & 48,132 & 141 & 99 & 72 \\
\hline Marquette Unfversity & 2 & 392 & 4,508 & 48,524 & 142 & 99 & 72 \\
\hline Montana State University & 2 & 304 & 4,510 & 48,828 & 143 & 99 & 73 \\
\hline South Dakota School of Mines \& Tech & 2 & 201 & 4,512 & 49,029 & 144 & 99 & 73 \\
\hline University of Alabama-B1rmingham & 2 & 101 & 4,514 & 49,130 & 145 & 100 & 73 \\
\hline Undversity of Central Florida & 2 & 293 & 4,516 & 49,423 & 146 & 100 & 74 \\
\hline Undversity of Hawa1i-Manoa & 2 & 181 & 4,518 & 49,604 & 147 & 100 & 74 \\
\hline University of Nevada-Reno & 2 & 102 & 4,520 & 49,706 & 148 & 100 & 74 \\
\hline University of New Hampshire & 2 & 154 & 4,522 & 49,860 & 149 & 100 & 74 \\
\hline University of Texas-Dallas & 2 & 0 & 4,524 & 49,860 & 150 & 100 & 74 \\
\hline Dartmouth college & 1 & 101 & 4,525 & 49,961 & 151 & 100 & 74 \\
\hline George Mason University & 1 & 0 & 4,526 & 49,961 & 152 & 100 & 74 \\
\hline Lamar University & 1 & 82 & 4,327 & 50,043 & 153 & 100 & 74 \\
\hline Naval Postgraduate School & 1 & 8 & 4,528 & 50,051 & 154 & 100 & 74 \\
\hline New York University & 1 & 0 & 4,529 & 50,051 & 155 & 100 & 74 \\
\hline Santa Clara University & 1 & 128 & 4,530 & 50,179 & 156 & 100 & 75 \\
\hline SUNY-Binghamton & 1 & 58 & 4,531 & 50,237 & 157 & 100 & 75 \\
\hline $\begin{array}{l}\text { University of California-San Francisco } \\
\text { University of Lowell }\end{array}$ & $\begin{array}{l}1 \\
1\end{array}$ & $\begin{array}{r}0 \\
381 \\
\end{array}$ & $\begin{array}{l}4,532 \\
4,533 \\
\end{array}$ & $\begin{array}{l}50,237 \\
50,618 \\
\end{array}$ & $\begin{array}{l}158 \\
159\end{array}$ & $\begin{array}{l}100 \\
100\end{array}$ & $\frac{75}{75}$ \\
\hline University of Southwestern Loulsiana & 1 & 207 & 4,534 & 50,825 & 160 & 100 & 76 \\
\hline University of Texas-HIth Sc1 Cntr Hston & 1 & 0 & 4,535 & 50,825 & 161 & 100 & 76 \\
\hline University of Hisconsin-Milwaukes & 1 & 171 & 4,536 & 50,996 & 162 & 100 & 76 \\
\hline California Poly st $U$, San Luis Obispo & 0 & 562 & & 51,558 & 163 & & 77 \\
\hline University of Puerto Rico-Mayaguez Cam & 0 & 518 & & 52,076 & 164 & & 77 \\
\hline GMI Engtneering and Management Inst itute & 0 & 505 & & 52,581 & 165 & & 78 \\
\hline Calfornia State Polytech Undv, Pomona & 0 & 435 & & 53,016 & 166 & & 79 \\
\hline California State Univ, Long Beach & 0 & 407 & & 53,423 & 167 & & 79 \\
\hline US Naval Academy & 0 & 399 & & 53,822 & 168 & & 80 \\
\hline CUNY-City College & 0 & 329 & & 54,151 & 169 & & 81 \\
\hline US Air Force Academy & 0 & 312 & & 54,463 & 170 & & 81 \\
\hline San Dlego State University & 0 & 305 & & 54,768 & 171 & & 81 \\
\hline San Jose State University & 0 & 288 & & 55,056 & 172 & & 82 \\
\hline Manhattan College & 0 & 280 & & 55,336 & 173 & & 32 \\
\hline California State Univ, Sacramento & 0 & 270 & & 55,606 & 174 & & 83 \\
\hline Temple University & 0 & 268 & & 55,874 & 175 & & 83 \\
\hline University of Wiscons1n-Platterille & 0 & 267 & & 56,141 & 176 & & 84 \\
\hline Rochester Institute of Technology & 0 & 260 & & 56,401 & 177 & & 84 \\
\hline California state Unlv, Fullerton & 0 & 232 & & 56,633 & 178 & & 84 \\
\hline Lawrence Institute of Technology & 0 & 228 & & 56,861 & 179 & & 85 \\
\hline Villanova University & 0 & 215 & & 57,076 & 180 & & 85 \\
\hline
\end{tabular}


Rose-Hulman Institute of Technology New York Institute of Technology

Callfornia State Univ, Fresno

Southern Illinois University-Carbondale

California State Univ, Ch1co

Wright State University

Milwaukee School of Engineering

California State Univ, Northridge

Memphis State Univergity

South Dakota State Univers Ity

Western Michlgan University

ITT Technical Institute-Indianapolis

California State Univ, Los Angeles

West Virglnia Ingtitute of Technology

University of Toxas-El Paso

Widener Unlversity Pennsylvanla Campus

Bradley University

Southeastern Massachusetts University

Youngstown State University

Pri-State University

Florlda International University

North Carolina Agri \& Tech State Univ

Northrop University

Universidad Politecnica de Puerto Rico

Embry-Riddle Aeronautical Univeisity

University of New Haven

Union College (NY)

Texas A \& I University

Bucknell University

Pratt Institute

Lafayette College

Fairleigh-Dickinson University

University of Hartford

University of North Carolina-Charlotte Cooper Union

Southern Illinols Univ-Edwardsville

Southern University and A \& $M$ College

San Erancisco State University

University of North Dakota

Montana College of Mineral Scl \& Tech

Seattle University

University of Portland

University of New Orleans

University of the Pacific

Florida State University

University of South Alabama

University of Evansville

Kent State University-A1I Campuses

Prairie Vlew Agric \& Mech Univ

Maine Maritime Academy

Virginia Military Institute

Western New England College

SUNY-Maritime College

Northern Arizona University

Us Coast Guard Academy

University of Bridgeport

Indiana Univers Ity-All Campuses

Oh1o Northern University

Tennessee State University

US Merchant Marine Academy

Gannon University

Citadel Military College of S Carolina

University of Tennessee-Chattanooga

Christian Brothers College

Valparaiso Univergity

Gonzaga University

Tuskegee University

Univerglty of Alaska-Falrbanks

University of Maryland-Baltimore County

Hofstra University

\begin{tabular}{l|l}
\hline & \\
\hline & \\
\hline & \\
\hline & \\
\hline & \\
\hline & \\
\hline & \\
\hline
\end{tabular}

\begin{tabular}{l|l}
0 & \\
0 & \\
0 & \\
0 & \\
0 & \\
0 & \\
0 & \\
\hline 0 & \\
\hline 0 & \\
\hline 0 & \\
0 & \\
\hline 0 & \\
\hline 0 & \\
\hline 0 & \\
\hline 0 & \\
\hline 0 & \\
\hline 0 & \\
\hline 0 & \\
\hline 0 &
\end{tabular}

\begin{tabular}{l|l}
211 & \\
\hline 206 & \\
\hline 197 & \\
\hline 197 & \\
\hline 195 & \\
\hline
\end{tabular}

\begin{tabular}{|l|r|l|l}
\hline 57,493 & 182 & & \\
\hline 57,690 & 183 & & \\
\hline 57,887 & 184 & & \\
\hline 58,082 & 185 & &
\end{tabular}

58,273

58,461

183

170

167

163

160

154

143

136

134

131

128

128

122

121

118

116

116

114

113

111

109

108

107

106

104

103

100

158

\begin{tabular}{|c|}
58,644 \\
58,814
\end{tabular}

\begin{tabular}{|l|l}
58,981 & 190
\end{tabular}

\begin{tabular}{l}
59,147 \\
\hline 59,310
\end{tabular}

\begin{tabular}{|c|c|}
59,310 & 192 \\
59,470 & 193
\end{tabular}

\begin{tabular}{|l|l|}
59,470 & 193 \\
\hline 59,627 & 194
\end{tabular}

$\left|\begin{array}{l}59,781 \\ 59,932\end{array}\right|$

$+160$

60,075

60,211

60,477

\begin{tabular}{|l|l|}
\hline 60,608 & 201 \\
\hline 60,736 & 202 \\
\hline 60,864
\end{tabular}

60,864

60,987

61,109

61,230

61,349

$-61,583$

61,699

61,813

62,039

62,150

62,260

62,369

62,477

62,584
62,691

62,797
62,902

63,006

63,109

63,210

63,310

63,507

63,602

63,695

63,785

63,961

64,046

64,128

$\frac{64,207}{64,285}$

64,361

64,437

64,511
64,585

64,585
64,658

64,658

64,728

$\frac{64,797}{64,865}$

64,93

64,998

65,062

65,126

65,190

65,253

195
196
197
198
199
200
201
202
203

203

205

206

207

208

209

210

211

212

213

214

215

226

217

218

219

221

222

223

224

225

226

228

229

230

- 231

232

233

234

235

236

237

$-\frac{238}{239}$

240

241

242

$-\frac{243}{244}$

$-\frac{244}{245}$

245
246
247

\begin{tabular}{|r|r|}
\hline & 97 \\
\hline & 97 \\
\hline
\end{tabular}

\begin{tabular}{l|r}
249 & 97 \\
\hline
\end{tabular}

250

97 


\begin{tabular}{|c|c|c|c|c|c|}
\hline University of Texas-San Antonio & 0 & 61 & 65,314 & 251 & 97 \\
\hline Grove City College & 0 & 56 & 65,370 & 252 & 97 \\
\hline Saint Cloud State University & 0 & 49 & 65,419 & 253 & 97 \\
\hline University of the District of Columbia & 0 & 49 & 65,468 & 254 & 97 \\
\hline Bridgeport Engineering Institute & 0 & 47 & 65,515 & 255 & 97 \\
\hline Norwich University-Military Coll of VT & 0 & 47 & 65,562 & 256 & 98 \\
\hline Harvey Mudd College & 0 & 46 & 65,608 & 257 & 98 \\
\hline Loyola Marymount University & 0 & 46 & 65,654 & 258 & 98 \\
\hline Walla Walla College & 0 & 46 & 65,700 & 259 & 98 \\
\hline Calvin College & 0 & 45 & 65,745 & 260 & 98 \\
\hline Geneva College & 0 & 45 & 65,790 & 261 & 98 \\
\hline University of Nevada-Ias Vegas & 0 & 44 & 65,834 & 262 & 98 \\
\hline Miami University-All Campuses $(\mathrm{OH})$ & 0 & 43 & 65,877 & 263 & 98 \\
\hline University of Californla-Santa Cruz & 0 & 43 & 65,920 & 264 & 98 \\
\hline West Coast University & 0 & 42 & 65,962 & 265 & 98 \\
\hline Northern Illinois University & 0 & 41 & 66,003 & 266 & 98 \\
\hline Wlikes College & 0 & 38 & 66,041 & 267 & 98 \\
\hline Saginaw valley state College & 0 & 36 & 66,077 & 268 & 98 \\
\hline Le Tourneau College & 0 & 32 & 66,109 & 269 & 98 \\
\hline Californda Martt ime Academy & 0 & 31 & 66,140 & 270 & 98 \\
\hline Merrimack College & 0 & 31 & 66,171 & 271 & 98 \\
\hline Mankato State University & 0 & 30 & 66,201 & 272 & 98 \\
\hline University of Wisconsin-Parkside & 0 & 29 & 66,230 & 273 & 99 \\
\hline MoNeese State Undversity & 0 & 28 & 66,258 & 274 & 99 \\
\hline Pactelc Lutheran University & 0 & 26 & 66,284 & 275 & 99 \\
\hline CUNY-College of Staten Island & 0 & 25 & 66,309 & 276 & 99 \\
\hline Idaho State University & 0 & 24 & 66,333 & 277 & 99 \\
\hline Monmouth College (NJ) & 0 & 24 & 66,357 & 278 & 99 \\
\hline University of Scranton & 0 & 24 & 66,381 & 279 & 99 \\
\hline Wentworth Institute of Technology (MA) & 0 & 24 & 66,405 & 280 & 99 \\
\hline Marietta College & 0 & 23 & 66,428 & 281 & 99 \\
\hline Colorado Technical College & 0 & 22 & 66,450 & 282 & 99 \\
\hline Southwest Texas State University & 0 & 22 & 66,472 & 283 & 99 \\
\hline University of Georgia & 0 & 22 & 66,494 & 284 & 99 \\
\hline Dowling College & 0 & 21 & 66,515 & 285 & 99 \\
\hline Morgan State University & 0 & 21 & 66,536 & 286 & 99 \\
\hline University of Alaska-Anchorage & 0 & 20 & 66,556 & 287 & 99 \\
\hline Webb Institute of Naval Architecture & 0 & 20 & 66,576 & 288 & 99 \\
\hline Arkansas State University-All Campuses & 0 & 19 & 66,595 & 289 & 99 \\
\hline Bay lor University & 0 & 19 & 66,614 & 290 & 99 \\
\hline Humboldt State University & 0. & 19 & 66,633 & 291 & 99 \\
\hline Swarthmore College & 0 & 19 & 66,652 & 292 & 99 \\
\hline Murray State University & 0 & 18 & 66,670 & 293 & 99 \\
\hline Saint Mary's University of San Antonio & 0 & 17 & 66,687 & 294 & 99 \\
\hline Loyola College & 0 & 16 & 66,703 & 295 & 99 \\
\hline Saint Martin's College & 0 & 16 & 66,719 & 296 & 99 \\
\hline Trinity College (CT) & 0 & 16 & 66,735 & 297 & 99 \\
\hline West virginia Wesleyan College & 0 & 14 & 66,749 & 298 & 99 \\
\hline Roosevelt University & 0 & 13 & 66,762 & 299 & 99 \\
\hline Saint Ambrose College & 0 & 13 & 66,775 & 300 & 99 \\
\hline Boise State University & 0 & 12 & 66,787 & 301 & 99 \\
\hline Mililkin Univergity & 0 & 12 & 66,799 & 302 & 99 \\
\hline Seattie Pacific University & 0. & 12 & 66,811 & 303 & 99 \\
\hline University of Redlands & 0 & 12 & 66,823 & 304 & 99 \\
\hline John Brown University & 0 & 11 & 66,834 & 305 & 99 \\
\hline Salem State College & 0 & 11 & 66,845 & 306 & 99 \\
\hline Trinity University & 0 & 11 & 66,856 & 307 & 99 \\
\hline University of wisconsin-stout & 0 & 11 & 66,867 & 308 & 99 \\
\hline Barry Univergity & 0 & 10 & 66,877 & 309 & 99 \\
\hline Fairfield University & 0 & 10 & 66,887 & 310 & 100 \\
\hline Hampton Untversity & 0 & 10 & 66,897 & 311 & 100 \\
\hline Hope College & 0 & 10 & 66,907 & 312 & 100 \\
\hline Oral Roberts University & 0 & 10 & 66,917 & 313 & 100 \\
\hline Philadelphia College of Textiles \& Scl & 0 & 10 & 66,927 & 314 & 100 \\
\hline SUNY-College at New Paltz & 0 & 10 & 66,937 & 315 & 100 \\
\hline University of Houston-Clear Lake & 0 & 10 & 66,947 & 316 & 100 \\
\hline Dordt College & 0 & 9 & 66,956 & 317 & 100 \\
\hline East Central Undversity & 0 & 9 & 66,965 & 318 & 100 \\
\hline
\end{tabular}




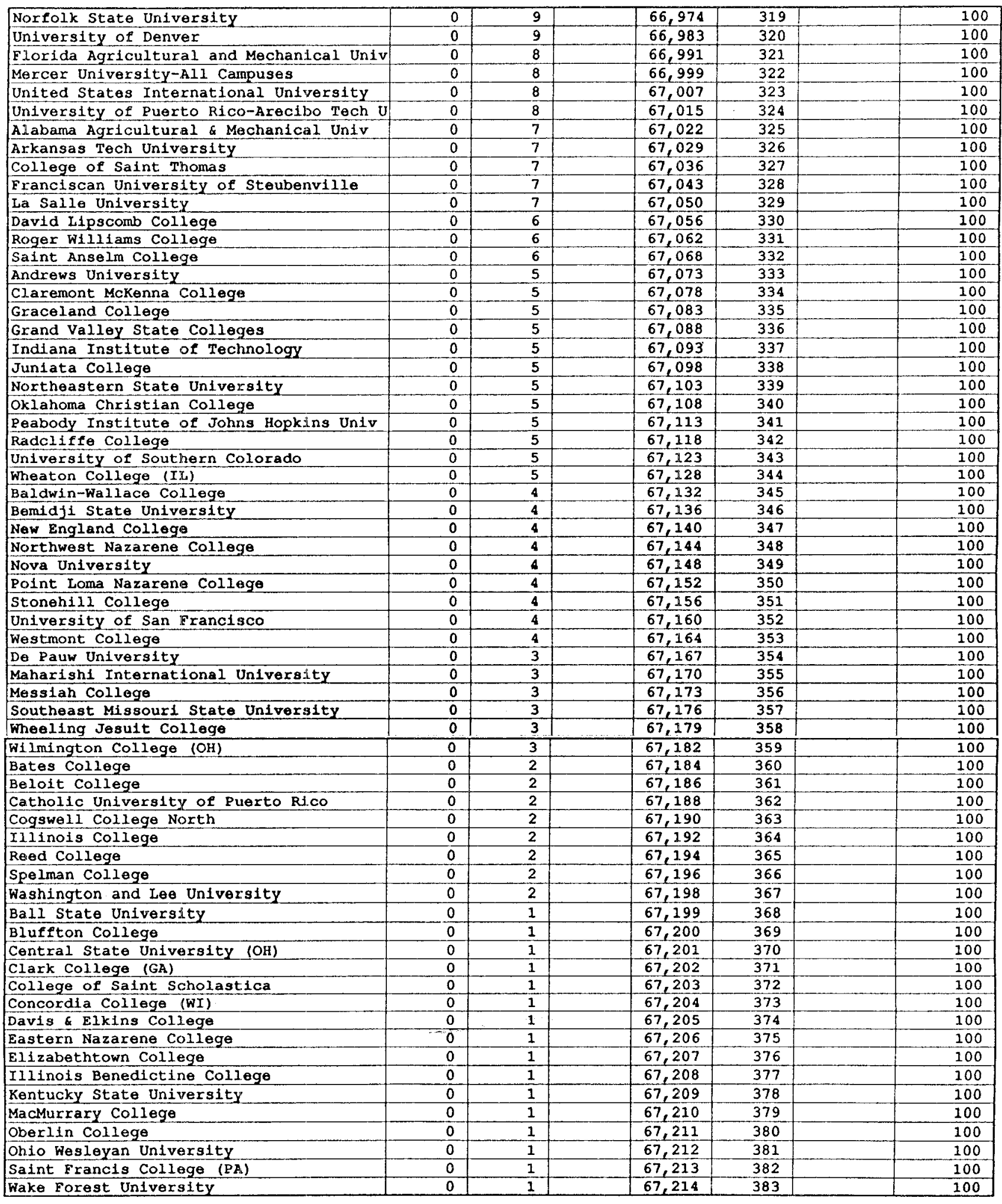


Figure I

Distribution of Engiteering Degrees in the United States, 1989

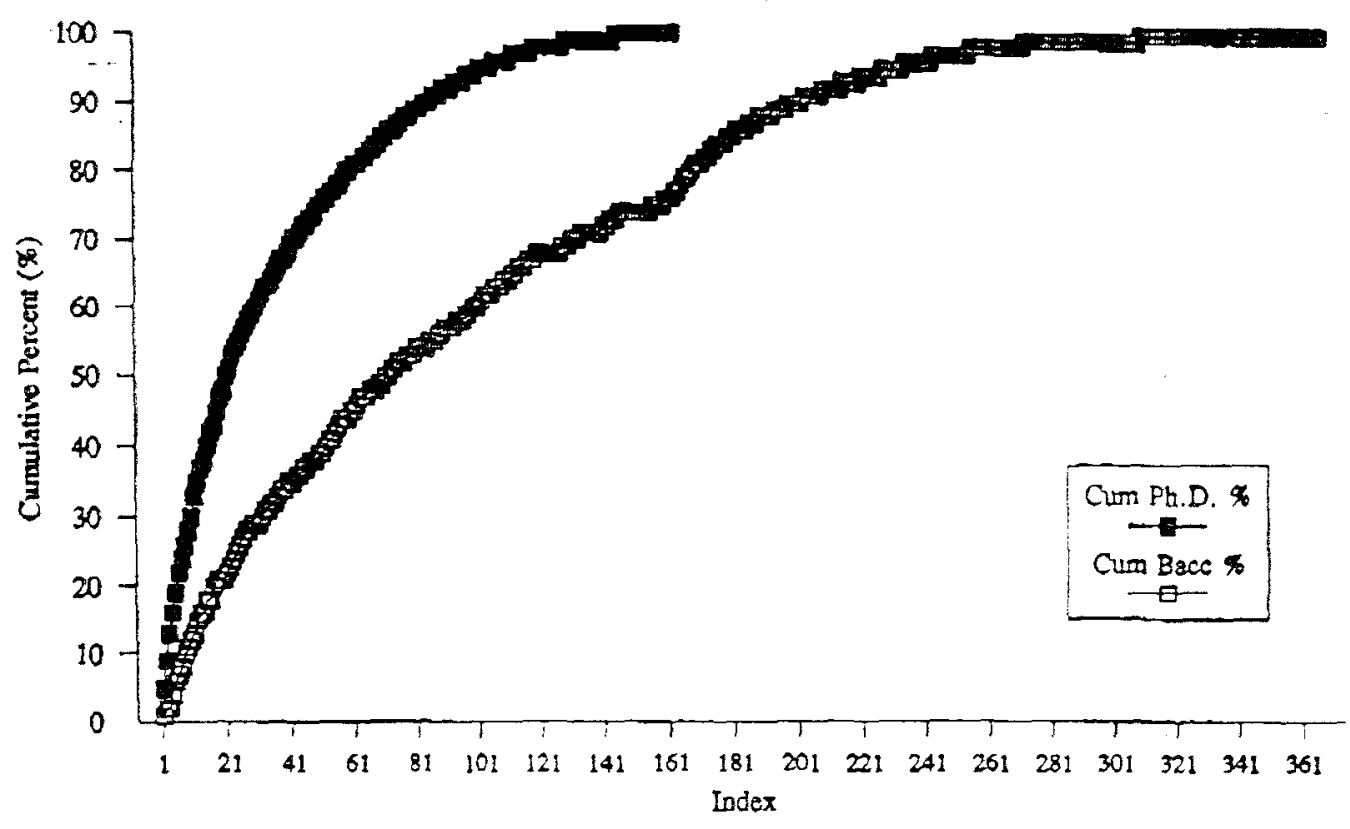


Engineering Ph.D.s and Bachelor's Degrees Conferred in California, by Institution: 1989 Table II

\begin{tabular}{|c|c|c|c|c|c|c|c|}
\hline Academic Institution & PhDs & Bach & Curn & Cum & Rank & Cum PhD : & Cum Bach 8 \\
\hline & & & $\mathrm{PhDs}$ & Bach & & 0 & 0 \\
\hline University of Californda-Berkeley & 201 & 517 & 201 & 517 & 1 & 30 & 8 \\
\hline Stanford University & 173 & 273 & 374 & 790 & 2 & 57 & 12 \\
\hline University of Southern California & 80 & 319 & 454 & 1,109 & 3 & 69 & 17 \\
\hline University of California-Los Angeles & 59 & 327 & 513 & 1,436 & 4 & 78 & 22 \\
\hline California Institute of Technology & 39 & 147 & 552 & 1,583 & 5 & 84 & 24 \\
\hline University of California-Davis & 35 & 372 & 587 & 1,955 & 6 & 89 & 30 \\
\hline Untversity of California-San Diego & 27 & 284 & 614 & 2,239 & 7 & 93 & 34 \\
\hline University of California-Santa Barbara & 23 & 194 & 637 & 2,433 & 8 & 96 & 37 \\
\hline University of Californla-Irvine & 21 & 181 & 658 & 2,614 & 9 & 100 & 40 \\
\hline Santa Clara University & 1 & 128 & 659 & 2,742 & 10 & 100 & 42 \\
\hline Naval Postgraduate School & 1 & 8 & 660 & 2,750 & 11 & 100 & 42 \\
\hline Unlvergity of Caltfornia-San Francisco & 1 & 0 & 661 & 2,750 & 12 & 100 & 42 \\
\hline California Poly st U, San Luis Oblspo & 0 & 562 & & 3,312 & 13 & & 50 \\
\hline Callfornia State Polytech Univ, Pomona & 0 & 435 & & 3,747 & 14 & & 57 \\
\hline California state Univ, Long Beach & 0 & 407 & & 4,154 & 15 & & 63 \\
\hline San Diego State University & 0 & 305 & & 4,459 & 16 & & 68 \\
\hline San Jose State University & 0 & 288 & & 4,747 & 17 & & 72 \\
\hline California State Univ, Sacramento & 0 & 270 & & 5,017 & 18 & & 76 \\
\hline California State unive Fullerton & 0 & 232 & & 5,249 & 19 & & 80 \\
\hline California State Univ, Fresno & 0 & 297 & & 5,446 & 20 & & 83 \\
\hline Caldfornia State Univ, Chico & 0 & 195 & & 5,641 & 21 & & 86 \\
\hline Callfornia State Untv, Northridge & 0 & 183 & & 5,824 & 22 & & 88 \\
\hline California State Univ, Los Angeles & 0 & 160 & & 5,984 & 23 & & 91 \\
\hline Northrop University & $\underline{0}$ & 128 & & 6,112 & 24 & & 93 \\
\hline San Francisco State Undversity & 0 & 107 & & 6,219 & 25 & & 94 \\
\hline University of the pacietc & 0 & 101 & & 6,320 & 26 & & 96 \\
\hline Harvey Mudd College & 0 & 46 & & 6,366 & 27 & & 97 \\
\hline Loyola Marymount University & 0 & 46 & & 6,412 & 28 & & 97 \\
\hline University of California-Santa Cruz & 0 & 43 & & 6,455 & 29 & & 98 \\
\hline West Coast University & 0 & 42 & & 6,497 & 30 & & 99 \\
\hline Californla Maritime Academy & 0 & 31 & & 6,528 & 31 & & 99 \\
\hline Humboldt State Universit:y & 0 & 19 & & 6,547 & 32 & & 99 \\
\hline University of Redlands & 0 & 12 & & 6,559 & 33 & & 100 \\
\hline United States International University & 0 & 8 & & 6,567 & 34 & & 100 \\
\hline Claremont McKenna Collegre & 0 & 5 & & 6,572 & 35 & & 100 \\
\hline Folnt Loma Nazarene College & 0 & 4 & & 6,576 & 36 & & 100 \\
\hline University of San Francisco & 0 & 4 & & 6,580 & 37 & & 100 \\
\hline Westmont college & 0 & 4 & & 6,584 & 38 & & 100 \\
\hline
\end{tabular}

Distribution of Engineering Degrees in California, 1989

Figure 2

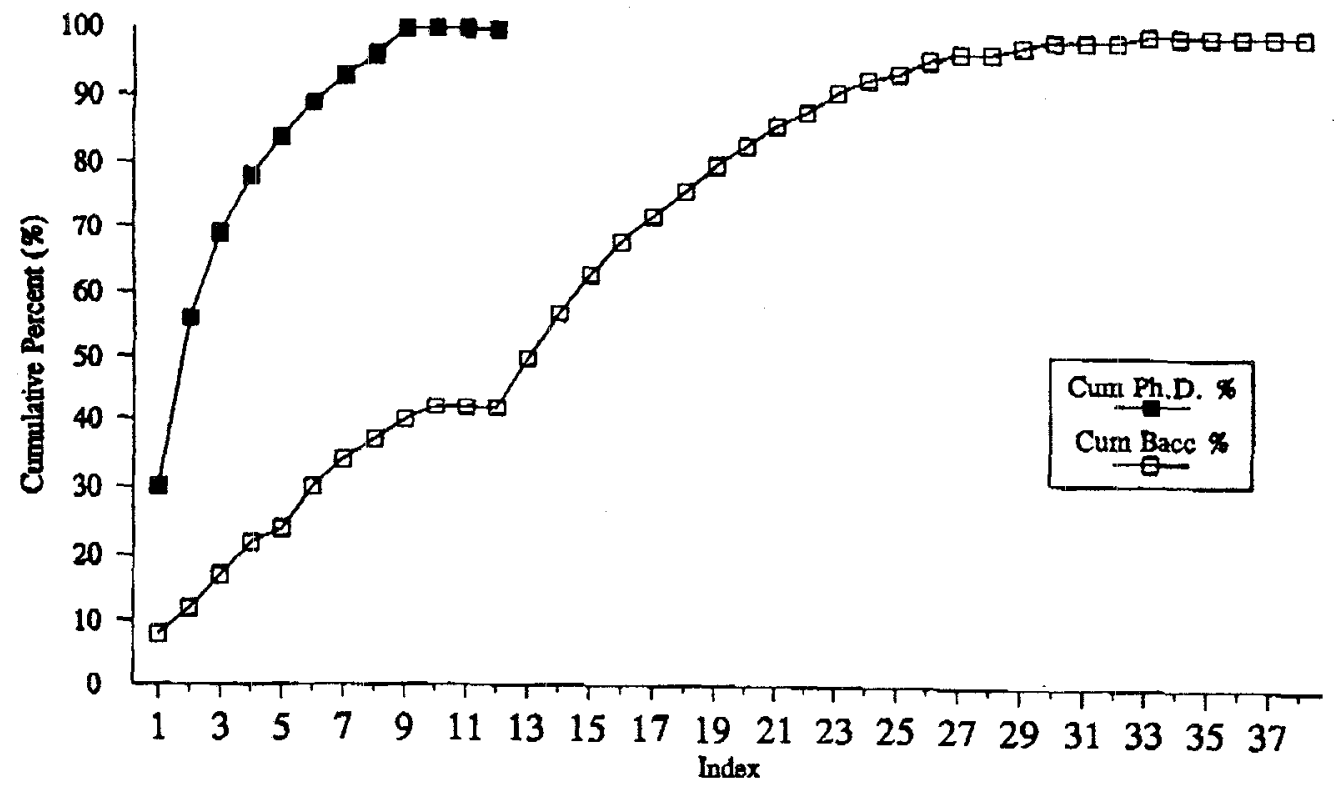


Englneering Ph.D.s and Bachelor's Degrees Conferred in Florida, by Institution: 1989

Table III

\begin{tabular}{|c|c|c|c|c|c|c|c|}
\hline Academic Institution & PhDs & Bach & Cum & Cum & Rank & Cum PhD & Cum Bach \\
\hline & & & PhDs & Bach & & 0 & 0 \\
\hline University of Florida & 60 & 583 & 60 & 583 & 1 & 71 & 26 \\
\hline Untversity of Miami (FL) & 9 & 266 & 69 & 849 & 2 & 81 & 37 \\
\hline University of South Florida & 6 & 380 & 75 & 1,229 & 3 & 88 & 54 \\
\hline Florida Atlantic University & 5 & 143 & 80 & 1,372 & 4 & 94 & 60 \\
\hline Florida Insitute of Technology & 3 & 237 & 83 & 1,609 & 5 & 98 & 71 \\
\hline University of Central Florida & 2 & 293 & 85 & 1,902 & 6 & 100 & 84 \\
\hline Florida International University & 0 & 131 & & 2,033 & 7 & & 89 \\
\hline Embry-RIddle Aeronautical University & 0 & 122 & & 2,155 & 8 & & 95 \\
\hline Florida state University & 0 & 100 & & 2,255 & 9 & & 99 \\
\hline Barry University & 0 & 10 & & 2,265 & 10 & & 99 \\
\hline Florida Agricultural and Mechanical Univ & 0 & 8 & & 2,273 & 11 & & 100 \\
\hline Nova University & 0 & 4 & & 2,277 & 12 & & 100 \\
\hline
\end{tabular}

Distribution of Engineering Degrees in Florida, 1989

Figure 3

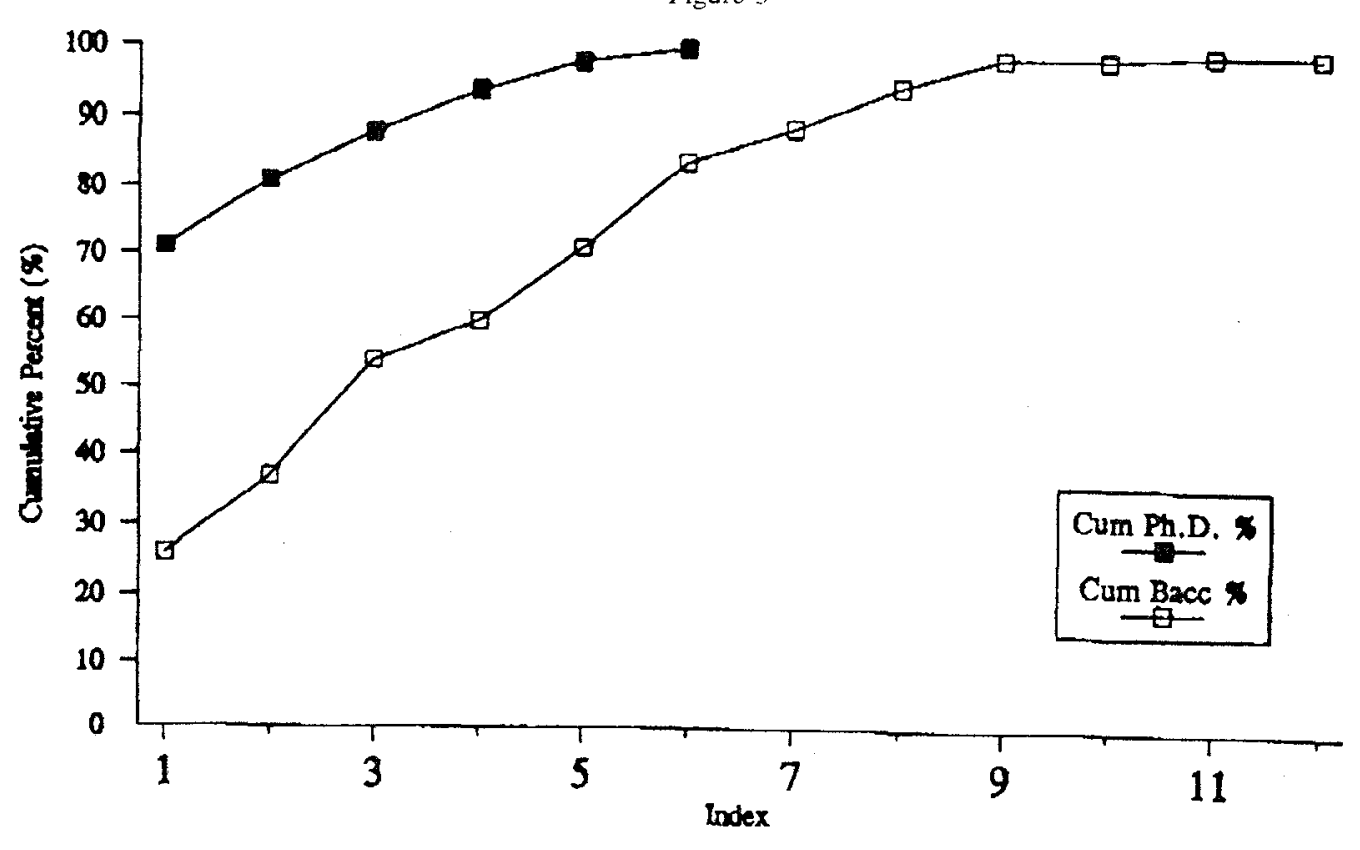


Englneering Ph.D.s and Bachelor's Degrees Conferred in rlino1s, by Inst:1tution: 1989

Table IV

\begin{tabular}{|c|c|c|c|c|c|c|c|}
\hline Academic Ingtitution & PnDs & Bach & Curn & Cum & Rank & Cum PhD & Cum $8 \mathrm{ach} 8$ \\
\hline & & & PnDs & Bach & & 0 & 0 \\
\hline Untversity of Illinols-Urbana & 136 & 1,259 & 136 & 1,259 & 1 & 54 & 46 \\
\hline Northwestern University & 74 & 250 & 210 & 1,509 & 2 & 83 & 55 \\
\hline University of Illinols-Chicago & 26 & 411 & 236 & 1,920 & 3 & 93 & 70 \\
\hline Ilinols Institute of Technology & 18 & 314 & 254 & 2,234 & 4 & 100 & 81 \\
\hline Southern Illinols University-Carbondale & 0 & 197 & & 2,431 & 5 & & 88 \\
\hline Bradiey University & 0 & 143 & & 2,574 & 6 & & 93 \\
\hline Southern Illinols Univ-Edwardsoille & 0 & 109 & & 2,683 & 7 & & 97 \\
\hline Northern Illinois University & 0 & 41 & & 2,724 & 8 & & 99 \\
\hline Roosevelt University & 0 & 13 & & 2,737 & 9 & & 99 \\
\hline Milixin University & 0 & 12 & & 2,749 & 10 & & 100 \\
\hline Wheaton College (II) & 0 & 5 & & 2,754 & 11 & & 100 \\
\hline Illinols college & 0 & 2 & & 2,756 & 12 & & 100 \\
\hline Ililnois Benedictine College & 0 & 1 & & 2,757 & 13 & & 100 \\
\hline MacMurrary College & 0 & 1 & & 2,758 & 14 & & 100 \\
\hline
\end{tabular}

Distribution of Engineering Degrees in Mlinois, 1989

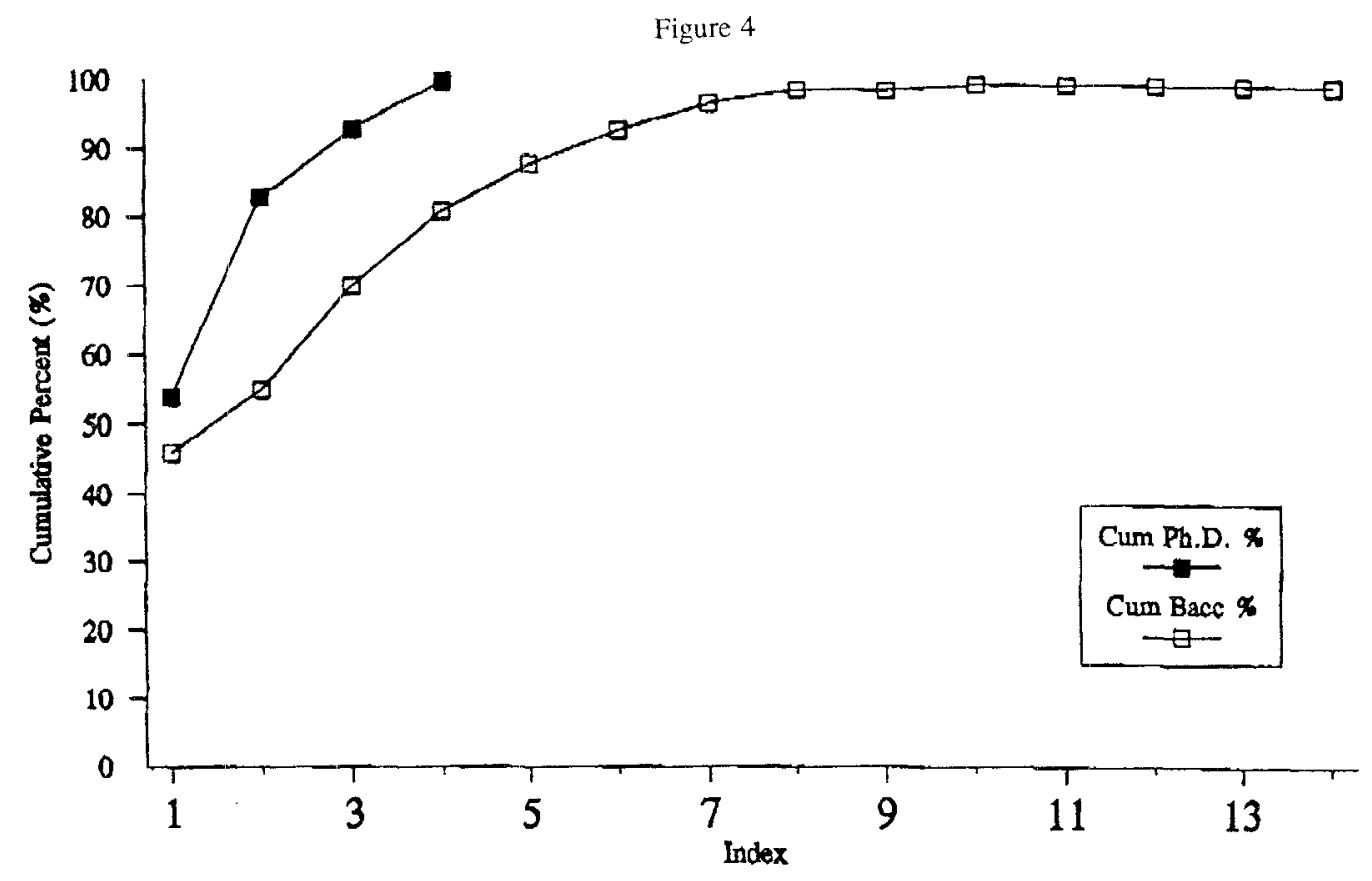


Engineering Ph.D.s and Bachelor's Degrees Conferred in Massachusetts, by Institution: 1989

Táble V

\begin{tabular}{|c|c|c|c|c|c|c|c|}
\hline Academic Institution & PhDs & $\mathrm{Bach}$ & Cum & Cum & Rank & Cum PhD & Cum Bach \\
\hline & & & PhDs & Bach & & 0 & 0 \\
\hline Massachugetts Institute of Technology & 217 & 633 & 217 & 633 & 1 & 75 & 19 \\
\hline Undversity of Massachusetts system & 43 & 418 & 260 & 1,051 & 2 & 89 & 32 \\
\hline Worcester Polytechnic Institute & 10 & 408 & 270 & 1,459 & 3 & 93 & 44 \\
\hline Northeastern University & 9 & 583 & 279 & 2,042 & 4 & 96 & 62 \\
\hline Harvard University & 4 & 27 & 283 & 2,069 & 5 & 97 & 63 \\
\hline Turts University & 4 & 158 & 287 & 2,227 & 6 & 99 & 68 \\
\hline Boston University & 3 & 385 & 290 & 2,612 & 7 & 100 & 79 \\
\hline University of Lowell & 1 & 381 & 291 & 2,993 & 8 & 100 & 91 \\
\hline Southeastern Massachusetts University & 0 & 136 & & 3,129 & 9 & & 95 \\
\hline Western New England College & 0 & 87 & & 3,216 & 10 & & 98 \\
\hline Merrimack College & 0 & 31 & & 3,247 & 11 & & 99 \\
\hline Wentworth Institute of Technology (MA) & 0 & 24 & & 3,271 & 12 & & 99 \\
\hline Salem State College & 0 & 11 & & 3,282 & 13 & & 100 \\
\hline Radcliffe College & 0 & 5 & & 3,287 & 14 & & 100 \\
\hline Stonehtll College & 0 & 4 & & 3,291 & 15 & & 100 \\
\hline Eastern Nazarene College & 0 & 1 & & 3,292 & 16 & & 100 \\
\hline
\end{tabular}

Distribution of Engincering Degrees in Massachusetts, 1989

Figure 5

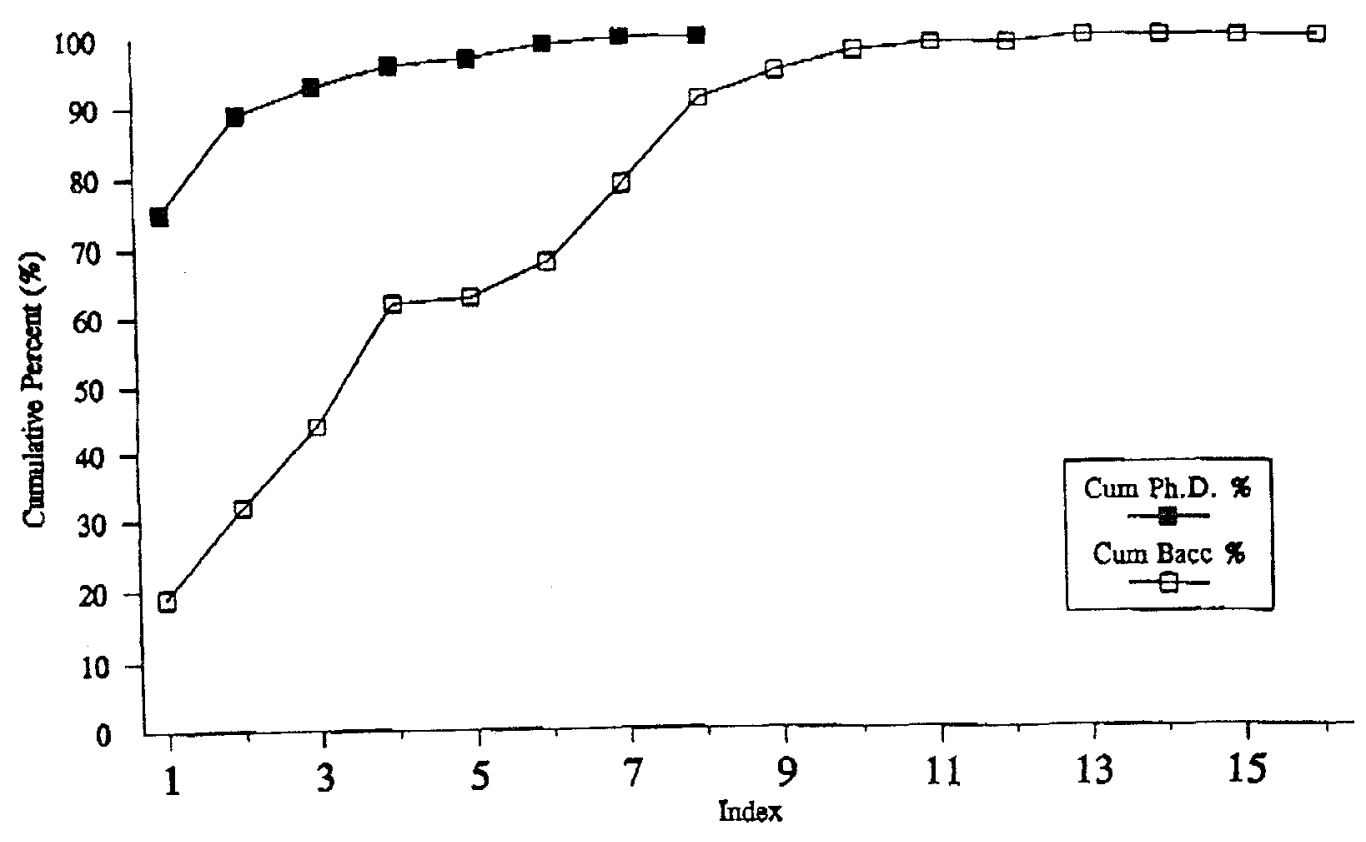


Englneering Ph.D.s and Eachelor's Degrees Conferred In Montana, by Institution: 1989 Table VI

\begin{tabular}{|c|c|c|c|c|c|c|c|}
\hline Academic Institution & PhDs & $\mathrm{Bach}$ & Cum & Cum & Rank & Cum PhD \& & Cum Bach \\
\hline & & & PhDg & Bach & & 0 & 0 \\
\hline Montana State University & 2 & 304 & 2 & 304 & 1 & 100 & 74 \\
\hline Montana College of Mineral Sci \& Tech & 0 & 106 & 2 & 410 & 2 & & 100 \\
\hline
\end{tabular}

Distribution of Engineering Degrees in Montana, 1989

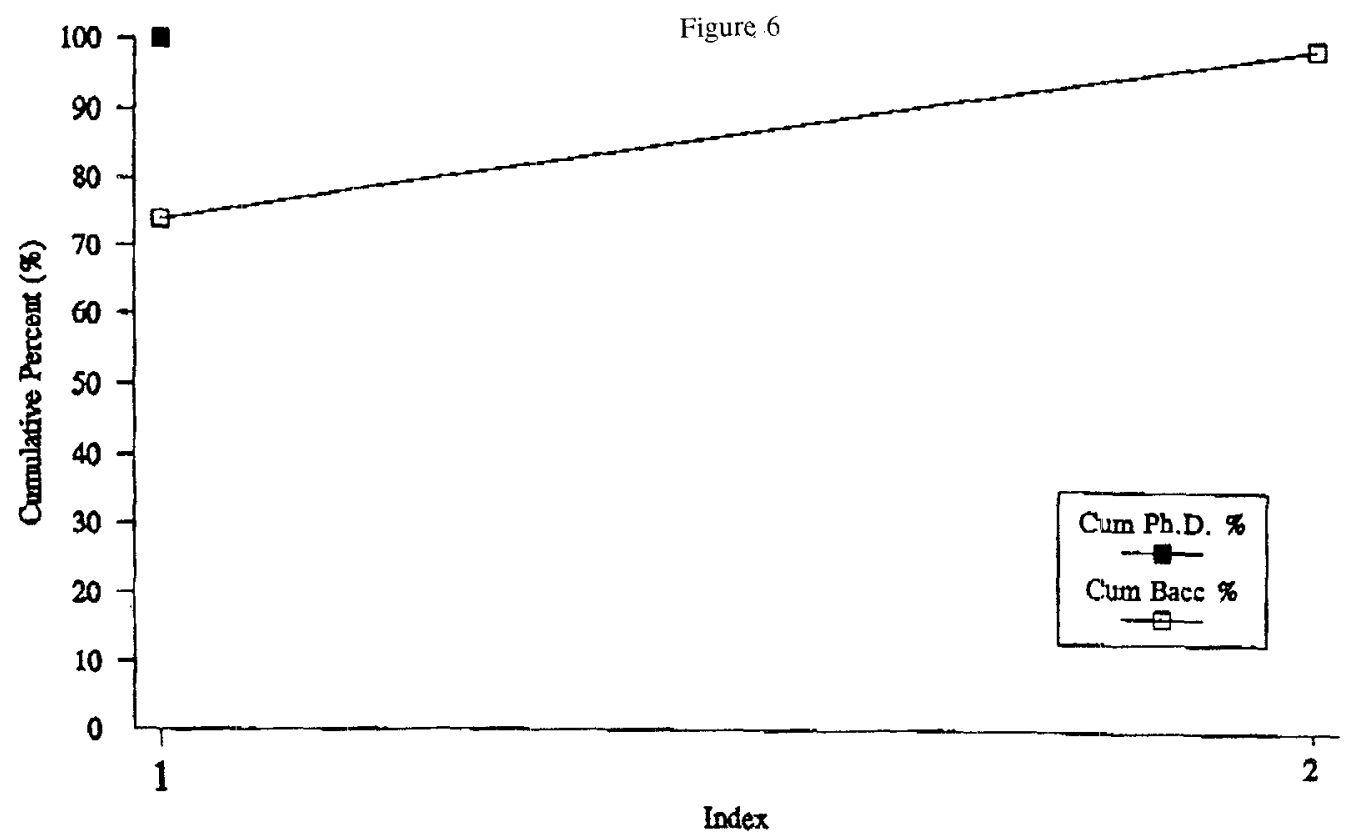


Engineering Fh.D.s and Bachelor's Degrees Conferred in New York, by Inst1tution: 1989

Table VII

\begin{tabular}{|c|c|c|c|c|c|c|c|}
\hline Academic Institution & PhDs & Bach & Cum & Cum & Rank & Cum PhD \& & Cum Bach 8 \\
\hline & & & PhDs & Bach & & 0 & 0 \\
\hline Corneli University & 88 & 568 & 88 & 568 & 1 & 25 & 10 \\
\hline Rensselaer polytechnic Institute & 73 & 618 & 161 & 1,186 & 2 & 45 & 22 \\
\hline Columbia University & 42 & 238 & 203 & 1,424 & 3 & 57 & 26 \\
\hline SUNY-Buffalo & 41 & 525 & 244 & 1,949 & 4 & 68 & 35 \\
\hline Clarkson University. & 25 & 659 & 269 & 2,608 & 5 & 75 & 47 \\
\hline Syracuse Unfversity-AIl Campuses & 24 & 239 & 293 & 2,847 & 6 & 82 & 52 \\
\hline Polytechnic University & 19 & 413 & 312 & 3,260 & 7 & 87 & 59 \\
\hline University of Rochester & 15 & 179 & 342 & 3,626 & 9 & 95 & 66 \\
\hline CuNY-Graduate School and University Cntr & 8 & 0 & 350 & 3,626 & 10 & 97 & 66 \\
\hline Alfred University-Main Campus & 5 & 89 & 355 & 3,715 & 11 & 99 & 68 \\
\hline Albany Law School union Universtty & 2 & 0 & 357 & 3,715 & 12 & 99 & 68 \\
\hline New York University & 1 & 0 & 358 & 3,715 & 13 & 100 & 68 \\
\hline SUNY-Binghamton & 1 & 58 & 359 & 3,773 & 14 & 100 & 69 \\
\hline CuNY-CIty College & & 329 & & 4,102 & 15 & & 75 \\
\hline Manhattan College & & 280 & & 4,382 & 16 & & 80 \\
\hline New York Institute of Technology & & 206 & & 4,848 & 18 & & 88 \\
\hline Union College (NY) & & 119 & & 4,967 & 19 & & 90 \\
\hline Pratt Institute & & 116 & & 5,083 & 20 & & 93 \\
\hline Cooper Union & & 110 & & 5,193 & 21 & & 95 \\
\hline SUNY-Maritime College & & 85 & & 5,278 & 22 & & 96 \\
\hline US Merchant Marine Acadeny & & 74 & & 5,352 & 23 & & 97 \\
\hline Hofstra University & & 63 & & 5,415 & 24 & & 99 \\
\hline CUNY-College of staten Island & & 25 & & 5,440 & 25 & & 99 \\
\hline Dowling College & & 21 & & 5,461 & 26 & & 99 \\
\hline Webb Institute of Naval Architecture & & 20 & & 5,481 & 27 & & 100 \\
\hline SUNY-College at New Paltz & & 10 & & 5,491 & 28 & & 100 \\
\hline
\end{tabular}

Distribution of Engineering Degrees in New York, 1989

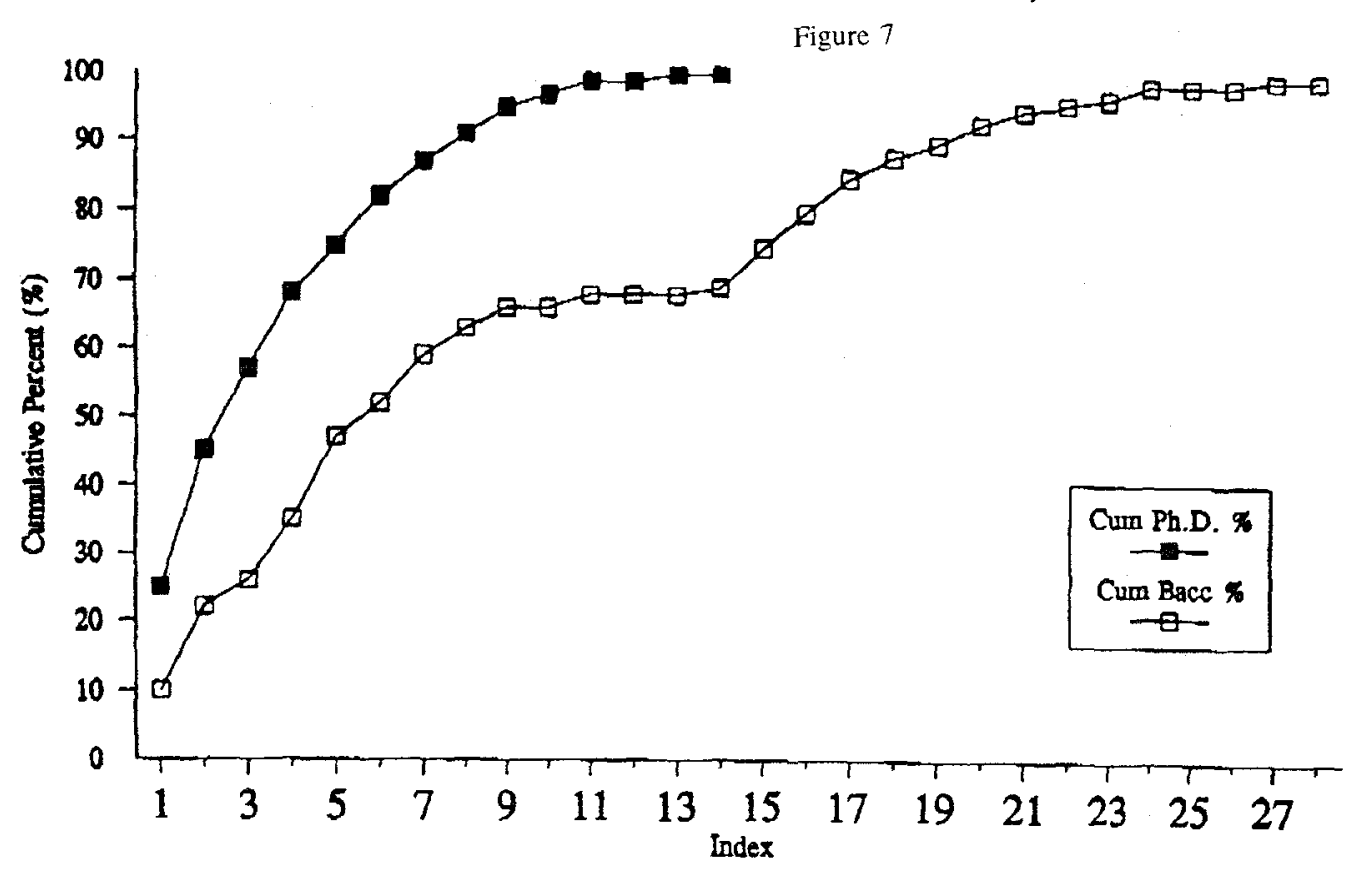


Engineerling Ph.D.s and Bachelor's Degrees Conferred in Texas, by Institution: 1989

Table VIII

\begin{tabular}{|c|c|c|c|c|c|c|c|}
\hline Academic Institution & PhDs & Bach & Cum & Cum & Rank & Cum PhD 8 & Cum Bach 8 \\
\hline & & & PhDs & Bach & & 0 & 0 \\
\hline University of Texas-Austin & 107 & 647 & 107 & 647 & 1 & 33 & 20 \\
\hline Texas $A \& M$ University-AII Campuses & 98 & 1,013 & 205 & 1,660 & 2 & 63 & 50 \\
\hline University of Houston & 32 & 202 & 237 & 1,862 & 3 & 73 & 56 \\
\hline Rice University & 27 & 145 & 264 & 2,007 & 4 & 82 & 61 \\
\hline Southern Methodist University & 21 & 91 & 285 & 2,098 & 5 & 88 & 64 \\
\hline University of Texas-Arlington & 18 & 340 & 303 & 2,438 & 6 & 94 & 74 \\
\hline Texas Tech University & 26 & 243 & 319 & 2,681 & 7 & 99 & 81 \\
\hline Lamar University & 1 & 82 & 322 & 2,763 & 9 & 100 & 84 \\
\hline University of Texas-HIth Sci Cntr Hston & 1 & 0 & 323 & 2,763 & 10 & 100 & 84 \\
\hline University of Texas-El Paso & 0 & 154 & & 2,917 & 11 & & 88 \\
\hline Texas A \& I University. & 0 & 118 & & 3,035 & 12 & & 92 \\
\hline Prairie View Agric \& Mech Univ & 0 & 93 & & 3,128 & 13 & & 95 \\
\hline University of Texas-San Antonio & 0 & 61 & & 3,189 & 14 & & 97 \\
\hline Le Tourneau College & 0 & 32 & & 3,221 & 15 & & 98 \\
\hline Southwest Texas state University & 0 & 22 & & 3,243 & 16 & & 98 \\
\hline Saint Mary's University of San Antonio & 0 & 17 & & 3,279 & 18 & & 99 \\
\hline Trinity University & 0 & 11 & & 3,290 & 19 & & 100 \\
\hline University of Houston-Clear lake & 0 & 10 & & 3,300 & 20 & & 100 \\
\hline
\end{tabular}

\section{Distribution of Engineering Degrees in Texas, 1989}

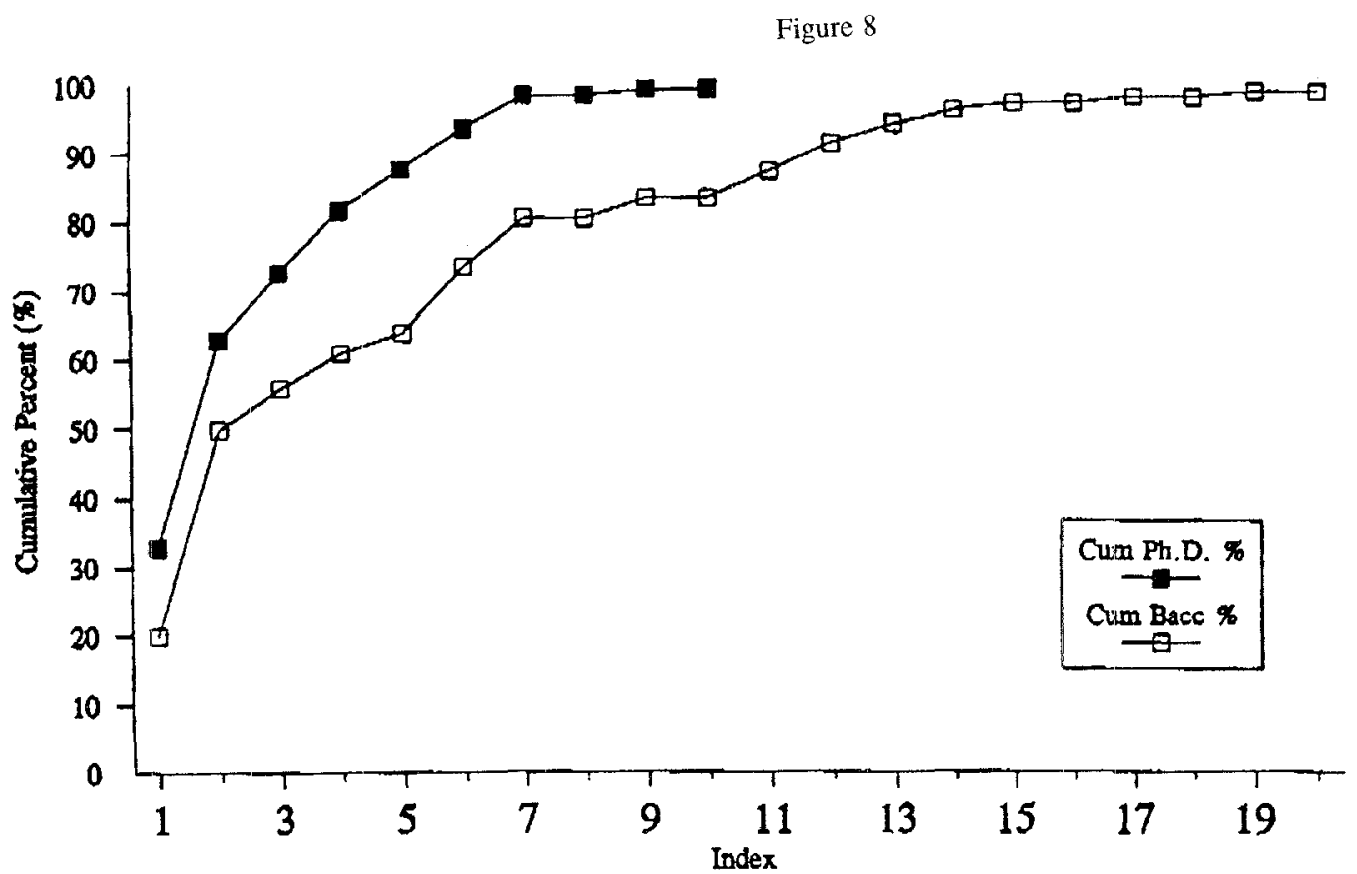




\section{James G. Harris}

James G. Harris received the B.S. and M.S. from UC-Berkeley in 1961 and 1962, and the Ph.D. from Syracuse University in 1968, all degrees in E.E. He taught at Howard University and the University of the District of Columbia, and worked at TRW in Redondo Beach, CA. He is a Professor at Cal Poly-SLO in the Electronic and Electrical Engineering Department, where he served as Head. He just completed two years as a Program Director at the National Science Foundation in the Division of Undergraduate Education. He is a member of IEEE, SIAM, ACM, AAAS, and ASEE.

\section{Win Aung}

Since joining the National Science Foundation in 1974, Win has held a number of responsible positions with the agency. He currently serves as Senior Staff Associate in the Division of Engineering Education and Centers, and works with programs dealing with research and education. Active in professional society service, he is a ASME Council on Education representative and member, Board on Research and Technology Development, and is chairman of the AIChE Field Committee on Emerging Technology. He was cochairman of the 1992 Engineering Foundation Conference on Engineering Education: Curriculum Innovation and Integration, and chairman of the 1990 International Symposium on Materials Processing and Manufacturing. $\mathrm{He}$ is an adjunct professor at the University of Maryland and Howard University where he teaches and carries out research. He has published over 100 technical articles and edited six books. Selected as the NSF Federal Engineer of the Year for 1985, Win is a Fellow of ASME and a registered Professional Engineer in the District of Columbia.

\section{Karen Frair}

Karen Frair is currently the Associate Dean for Administration in the College of Engineering at the University of Alabama. She received her B.S. from the University in 1967 and her M.S. and PhD degrees from the University of Oklahoma in 1972 and 1974 , all degrees in M.E. She was a member of the faculty in the Department of Engineering Science \& Mechanics at VPI\&SU, served as Associate Dean for Academic Affairs at Fresno State University, and was a Program Director in the Division of Undergraduate Education at NSF during the 1991-92 academic year. She is an Associate Editor of the Journal of Engineering Education, and is listed in American Men and Women of Science.

\section{Susan T. Hill}

Susan Hill is a statistician who has worked with Federal education data bases for almost 20 years. She did her undergraduate work at University of Maryland, College Park and her graduate work at George Washington University, Washington, D.C. She has been at the National Science Foundation since 1988 and has published reports on degree production in science/engineering fields. 